\title{
Experimental study of particle size segregation in 2D granular materials under vertical vibrations
}

\author{
Pawarut Jongchansitto $^{{ }^{*}}$, Thuwachit Kanyalert $^{1}$, and Itthichai Preechawuttipong ${ }^{1}$ \\ ${ }^{1}$ Department of Mechanical Engineering, Faculty of Engineering, Chiang Mai University, 239 Huay Kaew Rd., Muang District, Chiang \\ Mai 50200, Thailand
}

\begin{abstract}
The influence of particle size on segregation phenomena in two-dimensional binary granular mixtures with a similar solid fraction under vertical vibrations is experimentally investigated in this study. Four different particle size ratios $\left(D_{l} / D_{s}\right)$ are varied. The granular mixtures are subjected to vertical vibrations under six different dimensionless accelerations $(\Gamma=1-6)$, with constant vibrational amplitude of 5 $\mathrm{mm}$. The vertical segregation coefficient $(H)$ is here introduced to analyse the degree of segregation of the mixtures. We found that the Brazil nut effect, where the larger particle rises to the top, is observed for all the tests whenever the segregation is occurred. The degree of segregation of the mixtures is a function of the coefficient $H$, which can be divided into 3 zones: a compaction zone with particle mixing $(0.0<H \leq 0.2)$, a partially segregated zone $(0.2<H \leq 0.4)$, and an apparently segregated zone $(0.4<H \leq 1.0)$. The segregation begins when $\Gamma \geq 2$ and then tends to increase when $\Gamma$ is increased. In addition, the segregation is quite clearly found when $D_{l} / D_{s} \geq 3$ and $\Gamma \geq 3$.
\end{abstract}

\section{Introduction}

Since granular media are composed of grains with various physical properties such as size, density, friction and rigidity, complex behaviours of granular media are generally observed. These behaviours are cannot be simply classified into common substances [1,2]. In addition, such complex behaviours produce an extensive variety of phenomena that remains only partly understood.

"Segregation" is one of the interesting phenomena observed when a homogeneous bulk solid constituted from a variety of species or components evolves from an initially mixed state to a presumed non-uniform state owing to the relative movement within the materials [3, 4]. This is an unwanted phenomenon often occurring in many industrial areas associated with the handling and processing of granular mixtures such as pharmaceuticals, food processing, geophysics, material sciences, and almost all engineering fields. In fact, it must be realized that a complete particle mixing is the major purpose required in many industrial processes, rather than the unwanted segregation of the mixtures [3-6]. Although we have accepted for a long time that the segregation phenomena in granular media occurs from a difference of their physical properties (e.g. size, density, shape, etc.), a significant factor to control segregation in many industrial processes is the particle size [3, 5-7].

To better understand this problem, the present work aims at experimentally investigating the influence of particle size on the segregation phenomena in 2D granular binary mixtures, which are composed of two species of cylinder differing in diameter size but similar solid fraction, under vertical vibrations. Note that the solid fraction in 2D representation is defined by the ratio of the total particle area to the total container area. The effect of a dimensionless acceleration $(\Gamma)$, which governs the level of vibrations, is also studied.

\section{Experimental setup}

A commercial wooden cylinder with a density of about $404 \mathrm{~kg} / \mathrm{m}^{3}$ was used as a sample particle. The length of the cylinder is approximately $60 \mathrm{~mm}$ [8]. Four different ratios between large particle size and small particle size $\left(D_{l} / D_{s}\right)$ were changed for each granular sample as presented in Table 1. The sample was prepared by mixing two species of particle size inside an acrylic container. Note that the particles were sprayed in several colours, in order to distinguish the particle species when experiments were carried out. From the table, it can be noted that the number of particle in each species is not the same, in order to achieve a similar solid fraction between them.

Each granular mixture was then subjected to vertical vibrations provided by a cam-mechanism vibrational testing machine. Figure 1 presents an experimental setup in the present work. The level of the vibration was controlled by the dimensionless acceleration $(\Gamma)$, which is given by

$$
\Gamma=\frac{A \omega^{2}}{g}=\frac{A(2 \pi f)^{2}}{g}
$$

\footnotetext{
Corresponding author: pawarut.j@,cmu.ac.th
} 
where $A$ is the vibrational amplitude, $f$ is the frequency, and $g$ is the gravitational force. In this study, the vibrational amplitude was fixed at $5.0 \mathrm{~mm}$, while the frequency was progressively changed for obtaining the values of $\Gamma=1-6$.

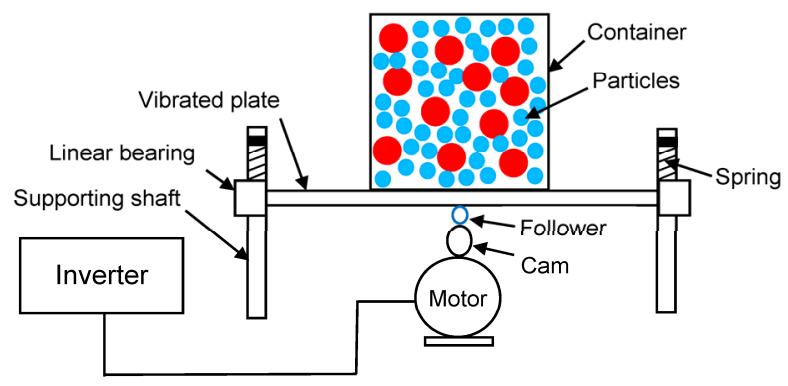

Fig. 1. Schematic drawing of an experimental setup

During the experiments, a photo was taken at each 30 s until reach final time at 420 s. Each photo was used for finding vertical positions of the centroid of each particle by using open-source software named "ImageJ" [9]. Specifying the vector $y_{i}$ as the height of particle $i$ with respect to the container base and the total number of particles of species inside the container is $N_{\alpha}$, the average height of particle can be thus determined by

$$
h_{\alpha}=\frac{1}{N_{\alpha}} \sum_{i=1}^{N_{\alpha}} y_{i}
$$

Table 1. Tested configurations

\begin{tabular}{|c|c|c|c|}
\hline \multirow{2}{*}{$\boldsymbol{D}_{\boldsymbol{l}} / \boldsymbol{D}_{\boldsymbol{s}}$} & $\begin{array}{c}\text { Diameter size } \\
(\mathbf{m m})\end{array}$ & $\begin{array}{c}\text { Number of } \\
\text { particles }\end{array}$ & Colours \\
\hline \multirow{2}{*}{2.0} & 12 & 246 & \\
\hline \multirow{2}{*}{3.0} & 10 & 350 & \\
\cline { 2 - 4 } & 12 & 500 & \\
\hline \multirow{2}{*}{3.0} & 12 & 56 & \\
\hline \multirow{2}{*}{4.0} & 4 & 500 & \\
\hline & 12 & 32 & \\
\hline & 3 & 500 & \\
\hline
\end{tabular}

These vertical positions were then employed to statistically compute the degree of segregation of the binary mixture in a static state, which is quantified by the vertical segregation coefficient $[10,11]$ :

$$
H=2 \frac{h_{l}-h_{s}}{h_{l}+h_{s}}
$$

where $h_{\alpha}$ is the average height of particles of species $\alpha=$ $l, s$. The factor 2 is a multiplication factor arising from the equal solid fraction of each particle species which limits the average height of particles in such species to $0.25<h_{\alpha}<0.75$ and thus $-1<H<1$. Note that the Brazil nut effect is initially observed when $H>0$, while the reverse Brazil nut effect found when $H<0$. The Brazil nut effect is segregation phenomena providing the upward movement of the larger particles under vibrated granular bed, contrary to the reverse Brazil nut effect. Figure 2 shows an example of a preparation of the granular mixture at an initial stage for all cases. In each $D_{l} / D_{s}$ ratio at the initial stage, the vertical segregation coefficient $(H)$ approaches to zero, which means that the system is homogeneous. In addition, it can be seen that although an initial configuration shown in Fig. 2a seems to be inhomogeneous, the value of the vertical segregation coefficient $(H)$ is nearly zero indicating the system is homogeneous.

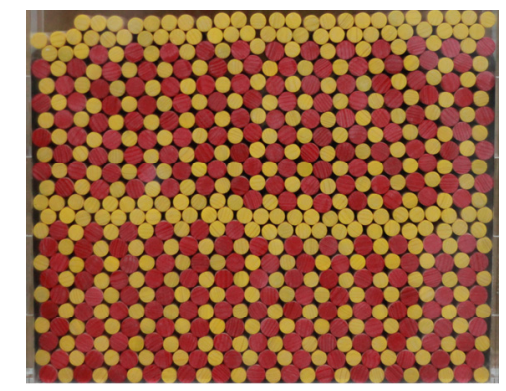

(a)

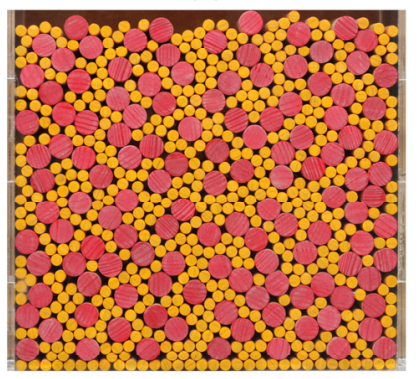

(b)

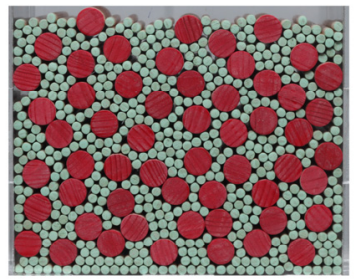

(c)

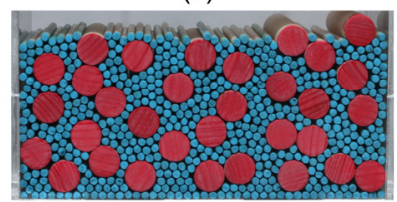

(d)

Fig. 2. Binary granular mixture at an initial stage in the case of: (a) $D_{l} / D_{s}=1.2$, (b) $D_{l} / D_{s}=2.0$, (c) $D_{l} / D_{s}=3.0$, and (d) $D_{l} / D_{s}=$ 4.0 under the level of vibration $\Gamma=5$ 


\section{Experimental results and discussions}

The influence of the particle size ratio $\left(D_{l} / D_{s}\right)$ and the dimensionless acceleration $(\Gamma)$ on segregation of the binary granular mixture is then described in this section by means of such vertical segregation coefficient.

The experimental results showed evidently that the Brazil nut effect is observed for all the samples which segregation happens. The vertical segregation coefficient (H) can be employed to characterize the degree of segregation of the mixture. In this case, this segregation degree can be split into 3 zones as follows. In the first zone with $0.0<H \leq 0.2$, the particles inside the binary mixture are still mixed, as shown in Fig. 3a. The applied vibration also makes the system more compacted. In the second zone, the binary granular mixture starts to segregate where $0.2<H \leq 0.4$ (see Fig. $3 \mathrm{~b}$ ), so that a partial segregation is observed here. In the third zone, segregation phenomena of the mixture are clearly apparently observed up to reach a complete segregation where $0.4<H \leq 1.0$, as displayed in Fig. 3c. (a)

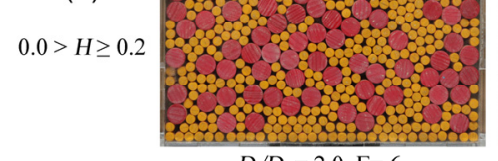

$D_{f} / D_{\mathrm{s}}=2.0, \Gamma=6$

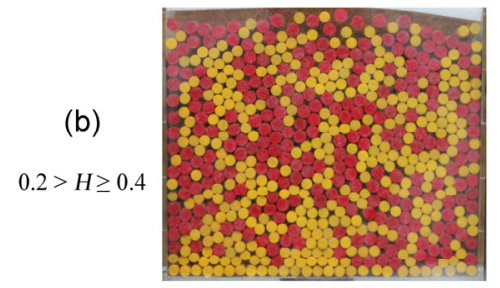

$D_{/} / D_{\mathrm{s}}=1.2, \Gamma=6$

(c)

$0.4>H \geq 1.0$

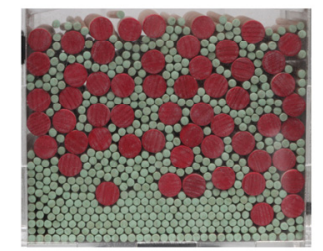

$D_{f} / D_{s}=3.0, \Gamma=6$

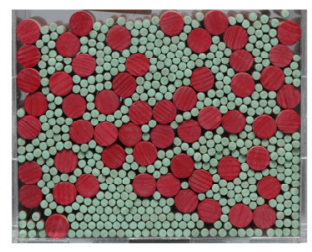

$D_{f} / D_{\mathrm{s}}=3.0, \Gamma=3$

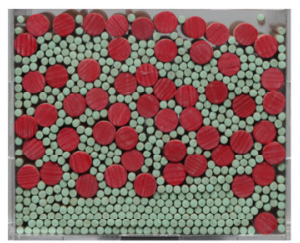

$D_{f} / D_{s}=3.0, \Gamma=5$

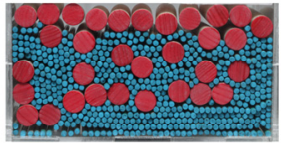

$D_{f} / D_{s}=4.0, \Gamma=5$

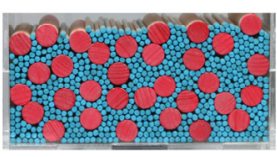

$D_{f} / D_{\mathrm{s}}=4.0, \Gamma=2$

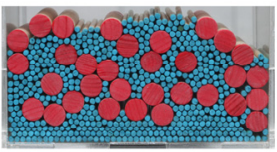

$D_{f} / D_{s}=4.0, \Gamma=3$

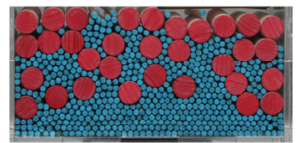

$D_{f} / D_{s}=4.0, \Gamma=6$

Fig. 3. Examples of segregation zones in different cases of $D_{l} / D_{s}$ ratio at $420 \mathrm{~s}$ under different levels of vibration: (a) $0.0<H \leq 0.2$, (b) $0.2<H \leq 0.4$, and (c) $0.4<H \leq 1.0$

\subsection{The effect of the particle size ratio}

Figure 4 presents the influence of particle size ratio $\left(D_{l} / D_{s}\right)$ on the segregation phenomena in the binary granular mixture. It is interesting to note that most values of the vertical segregation coefficient in the case of $D_{l} / D_{s}$ $=1.2$ are found in the first zone of segregation. In contrast, when the $D_{l} / D_{s}$ ratio is increased to be 3.0 and 4.0 , the segregation coefficient values are observed almost in the second zone and the third zone, respectively. It can be also observed that chance of segregation occurrence is higher when the $D_{l} / D_{s}$ ratio is increased. The maximum vertical segregation coefficient is equal to 0.56 , which occurs at $\Gamma=6$ in the case of $D_{l} / D_{s}=4.0$.

\subsection{The effect of the dimensionless acceleration}

The degree of segregation is plotted as a function of the dimensionless acceleration as shown in Fig. 5. No segregation occurs at $\Gamma=1$ even though we increase the
$D_{l} / D_{s}$ ratio. In this case, the binary granular mixture remains mixed. Furthermore, the mixture begins to segregate when $\Gamma \geq 2$ for all the particle size ratios. It is clearly seen that the vertical segregation coefficient increases for all the particle size ratios, when the dimensionless acceleration is increased.

From Figs. 4 and 5, it seems that there is something wrong observed in the experimental results. First, the vertical segregation coefficient in the case of $D_{l} / D_{s}=1.2$ at $\Gamma=2$ is higher than that of the other particle size ratios, despite the fact that its value observed when $\Gamma>2$ is the lowest, except for $D_{l} / D_{s}=2.0$. Second, the lowest vertical segregation coefficient is found in the case of $D_{l} / D_{s}=2.0$ when $\Gamma \geq 2$. These errors can be explained by the effect of container size that we did not consider carefully in the experimental setup. In other words, an aspect ratio between width and height $(w / h)$ of the container used for each case: the $w / h$ ratio for $D_{l} / D_{s}=2.0$ $>$ the $w / h$ ratio for $D_{l} / D_{s}=3.0$ and $4.0>$ the $w / h$ ratio for $D_{l} / D_{s}=1.2$. A difference of the container size may cause a different intensity of bulk convection motion, which is 
one of main mechanisms behind segregation phenomena $[6,12,13]$.

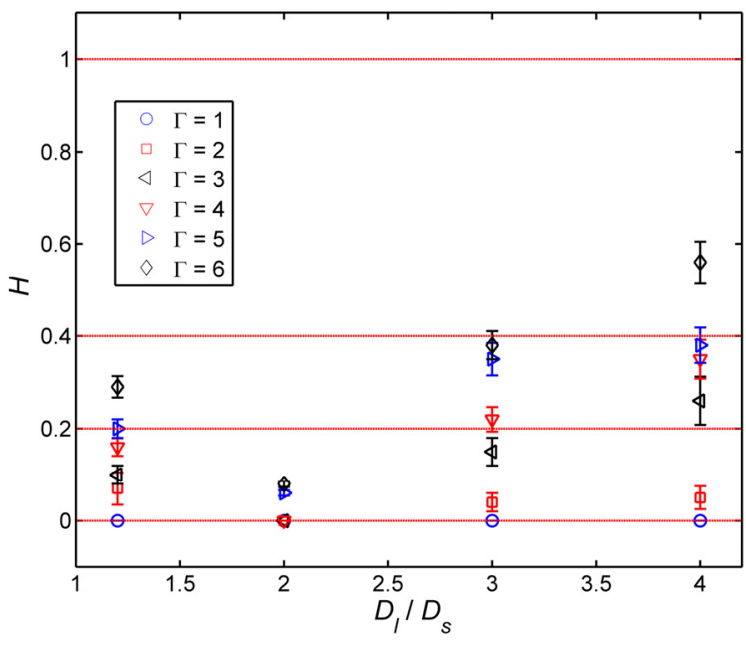

Fig. 4. The vertical segregation coefficient $(H)$ as a function of the ratio between large particle size and small particle size $\left(D_{l} / D_{s}\right)$ at $420 \mathrm{~s}$

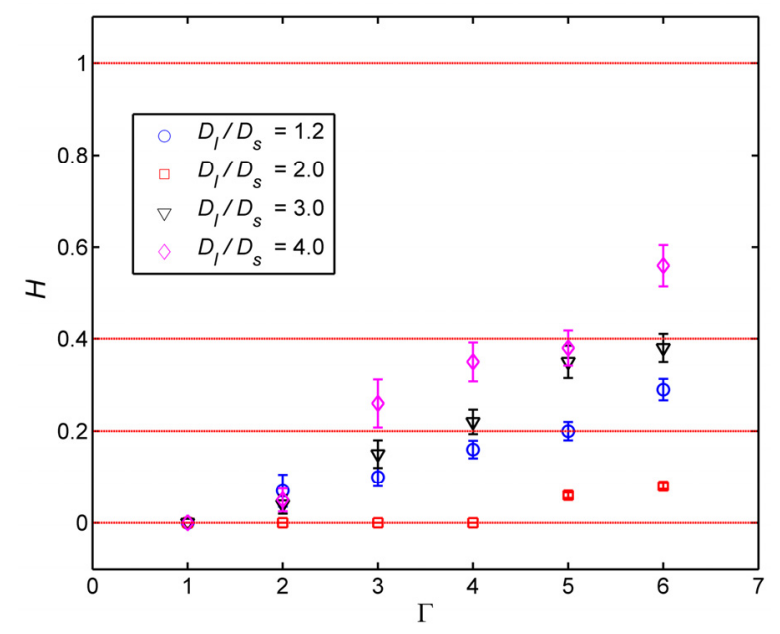

Fig. 5. The vertical segregation coefficient $(H)$ as a function of the dimensionless acceleration $(\Gamma)$ at $420 \mathrm{~s}$

\section{Conclusions}

The objective of this study is to experimentally investigate the effects of the particle size ratio $\left(D_{l} / D_{s}\right)$ and the dimensionless acceleration $(\Gamma)$ on granular segregation in the binary mixture subjected to vertical vibrations. Note that the solid fraction of each particle species is the same. It was found that for all the samples, the larger particle size migrates to the top of the container. This phenomenon is well-known as the Brazil nut effect. Based on the vertical segregation coefficient $(H)$, we can separate the degree of segregation of the mixture into 3 zones as follows. The particles are still in the mixed state at the first zone lying between $0.0<H \leq$ 0.2 . The particles then begin to segregate at the second zone where $0.2<H \leq 0.4$. In this zone, a partial segregation can be seen. The segregation of the mixture is more clearly observed up to obtain a complete segregation in the third zone where $0.4<H \leq 1.0$.
Interestingly, they start to segregate when $\Gamma \geq 2$ for all the particle size ratios. The vertical segregation coefficient increases with the dimensionless acceleration. The segregation of the mixture is quite clearly observed when $D_{l} / D_{s} \geq 3.0$. This segregation phenomenon is clearer when the particle size ratio is increased.

In a future work, it would be interesting to perform the numerical simulations based on the experimental configurations, in order to analyse between experimental and numerical results. Moreover, the simulations would be employed as a supplementary tool for investigating other cases that the experiments cannot be conducted due to their difficulties. Importantly, the effect of container size must be identified thoroughly for the next study.

\section{References}

1. H.M. Jaeger, S.R. Nagel, Science 255, 1523 (1992)

2. H.M. Jaeger, S.R. Nagel, Rev. Mod. Phys. 68, 1259 (1996)

3. A.D. Rosato, D.L. Blackmore, N. Zhang, Y. Lan, Chem. Eng. Sci. 57, 265 (2002)

4. D. Pihler-Puzović, T. Mullin, P. Roy. Soc. A 469, 20130203 (2003)

5. A.D. Rosato, Y. Lan, D.T. Wang, Powder Technol. 66, 149 (1991)

6. J.B. Knight, H.M. Jaeger, S.R. Nagel, Phys. Rev. Lett. 70, 3728 (1993)

7. J.C. Williams, Powder Technol. 15, 245 (1976)

8. F. Calvetti, G. Combe, J. Lanier, Mech. Cohes.Frict. Mat. 2, 121 (1997)

9. W.S. Rasband, ImageJ, (U.S. National Institutes of Health, Bethesda, Maryland, USA, http://imagej.nih.gov/ij/, 1997-2016)

10. C.R.A. Abreu, F.W. Tavares, M. Castier, Powder Technol. 134, 167 (2003)

11. M.P. Ciamarra, M.D. De Vizia, A. Fierro, M. Tarzia, A. Coniglio, M. Nicodemi, Phys. Rev. Lett. 96, 058001 (2006)

12. T. Pöschel, H.J. Herrmann, Europhys. Lett. 29, 123 (1995)

13. W. Cooke, S. Warr, J.M. Huntley, R.C. Ball, Phys. Rev. E 53, 2812 (1996) 